\title{
A cadaveric analysis of anatomical variations of the anterior belly of the digastric muscle
}

\author{
H. Anderson ${ }^{0}$, R.P. Tucker \\ Department of Cell Biology and Human Anatomy, University of California, Davis, United States
}

[Received: 2 June 2020; Accepted: 20 July 2020; Early publication date: 2 September 2020]

Background: The anterior belly of the digastric muscle (ABDM) presents highly variable and frequent anatomical variations. Since the ABDM functions as a landmark for clinical procedures involving the submental region, it is important to have a comprehensive understanding of its variations. In this study, we sought to improve our knowledge of ABDM variations in the ethnically diverse whole-body donor population in Northern California. Specific aims were: (1) to determine the frequency of $A B D M$ and anterior belly $(A B)$ accessory muscle variations in cadavers donated to the UC Davis Body Donation Programme, (2) to classify these variations identified in this population using the previously proposed nomenclatures by Yamada (1935) and Zlabek (1933), and (3) to investigate the innervation and arterial supply to the representative $A B D M$ and $A B$ accessory muscle variations. Materials and methods: During the 2018 and 2019 gross anatomy dissection laboratories at the UC Davis School of Medicine, the submental regions of 48 cadavers were examined and classified.

Results: Fifteen (31.2\%) cadavers presented ABDM and $A B$ accessory muscle variations. These variations were clearly categorised using the morphology of the $A B D M s$ and attachments of the $A B$ accessory muscles. We also identified three previously unreported types of variations, two of which presented the fusion of right and left $A B D M s$ and one presenting an ectopic tubercle beneath the mandibular symphysis to which a pair of $A B$ accessory muscles were attached. Conclusions: Anterior belly of the digastric muscle variations were found in 1 in 3 individuals in the local Northern California population. Knowledge of the prevalence and common patterns of ABDM variations in the general population would be valuable information when an operation or examination is performed in the submental region. (Folia Morphol 2021; 80, 3: 691-698)

Key words: anatomical variation, submental region, suprahyoid muscle, cadaver, gross anatomy laboratory

\section{INTRODUCTION}

The anterior belly of the digastric muscle (ABDM) typically originates from the digastric fossa of the mandible and inserts onto the body and greater horn of the hyoid bone and the intermediate tendon that it shares with the posterior belly of the digastric muscle. Variations in this arrangement were reported as early as in the $18^{\text {th }}$ century [25], in the $19^{\text {th }}$ century (e.g. [11]), and a broad range of variations were classified by Zlabek [28] and Yamada [25]. The frequency of ABDM 

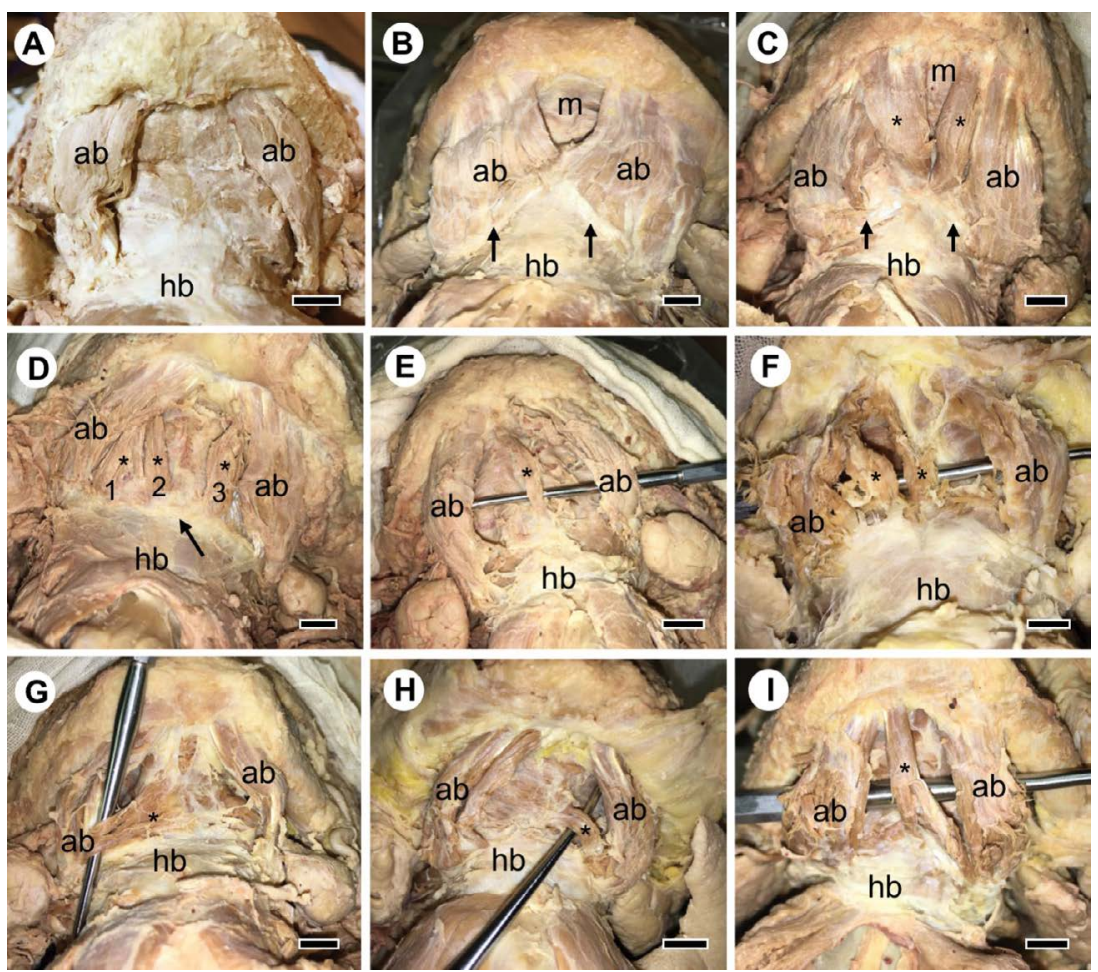

Figure 1. Summary of 1 standard and 8 variations of anterior belly of the digastric muscle (ABDM) observed in the 2018 gross anatomy laboratory. The age of each cadaver and brief description of the observed variations are summarised; $\mathbf{A}$. 76-year-old male presenting a standard pattern of the ABDM and the mylohyoid muscle; B. 62-year-old male with fused sheet-like ABDMs. Atavistic type; C. 55-year-old male with a pair of anterior belly $(A B)$ accessory muscles. Bilateral origin (anterior) type; D. 60-year-old male with three $A B$ accessory muscles (1-3). All three accessory muscles insert into the fibrous tendinous arch. Bilateral origin (anterior) type; $E$. 63-year-old male with an $A B$ accessory muscle on the right side. Unilateral origin (anterior) type; F. 85-year-old male with two $A B$ accessory muscles on the right side. Unilateral origin (anterior) type; G. 90-year-old female with an $A B$ accessory muscle on the right side. Unilateral insertion (posterior) type; H. 70-year-old male with an $A B$ accessory muscle on the left side. Unilateral insertion (posterior) type; I. 57-year-old female with an $A B$ accessory muscle traversing across the midline. The insertion side of the accessory muscle is bifurcated. Mixed type with variation of bifurcation; ab - anterior belly of the digastric muscle; hb — hyoid bone; asterisk — accessory anterior belly muscles; arrows — fibrous tendinous arch. The scale bar corresponds to $1 \mathrm{~cm}$.

anatomical variations in dissected cadavers reported in the literature differs significantly, ranging from $5 \%$ to $70 \%[8,13,17,25,28]$. These differences could be attributed to the small sample size in some studies as well as characteristics stemming from the common ancestry of the sample population. Types of structural variations reported for the ABDM and its accessory muscles also demonstrate significant diversity, including non-standard morphology of the ABDM itself, the location and number of anterior belly $(A B)$ accessory muscles, and relationships of $A B$ accessory muscles with the mylohyoid muscle $[3,8,25,28]$. These complexities present a challenge in categorising $A B D M$ variations.

In the current study we examined 48 cadavers for the incidence and types of variations in the ABDM and $A B$ accessory muscles. These variations were classified according to the schema proposed by Yamada (1935) [25] and Zlabek (1933) [28]. During the course of this investigation three previously undescribed variations were found, and the blood supply and innervation of the representative variants were investigated. Our study confirms that variations in the ABDM are common, and that many novel variations may remain to be described. These variations may be of interest to developmental biologists studying the origins of the suprahyoid muscles as well as to clinicians who perform examination and surgeries in the submental and digastric triangles of the neck.

\section{MATERIALS AND METHODS}

Forty-eight cadavers (male 23 , female 25 , average age 75 ) dissected by first year medical students in the gross anatomy laboratory over a 2-year period (2018 and 2019) at the UC Davis School of Medicine were included in this study. Bodies were originally donated to the UC Davis Body Donation Programme. During the dissection of the submental region in the laboratory, an atypical arrangement of the ABDM, being accompanied with bilateral accessory muscle bundles, was identified in 1 cadaver (Fig. 1C). This finding prompted 

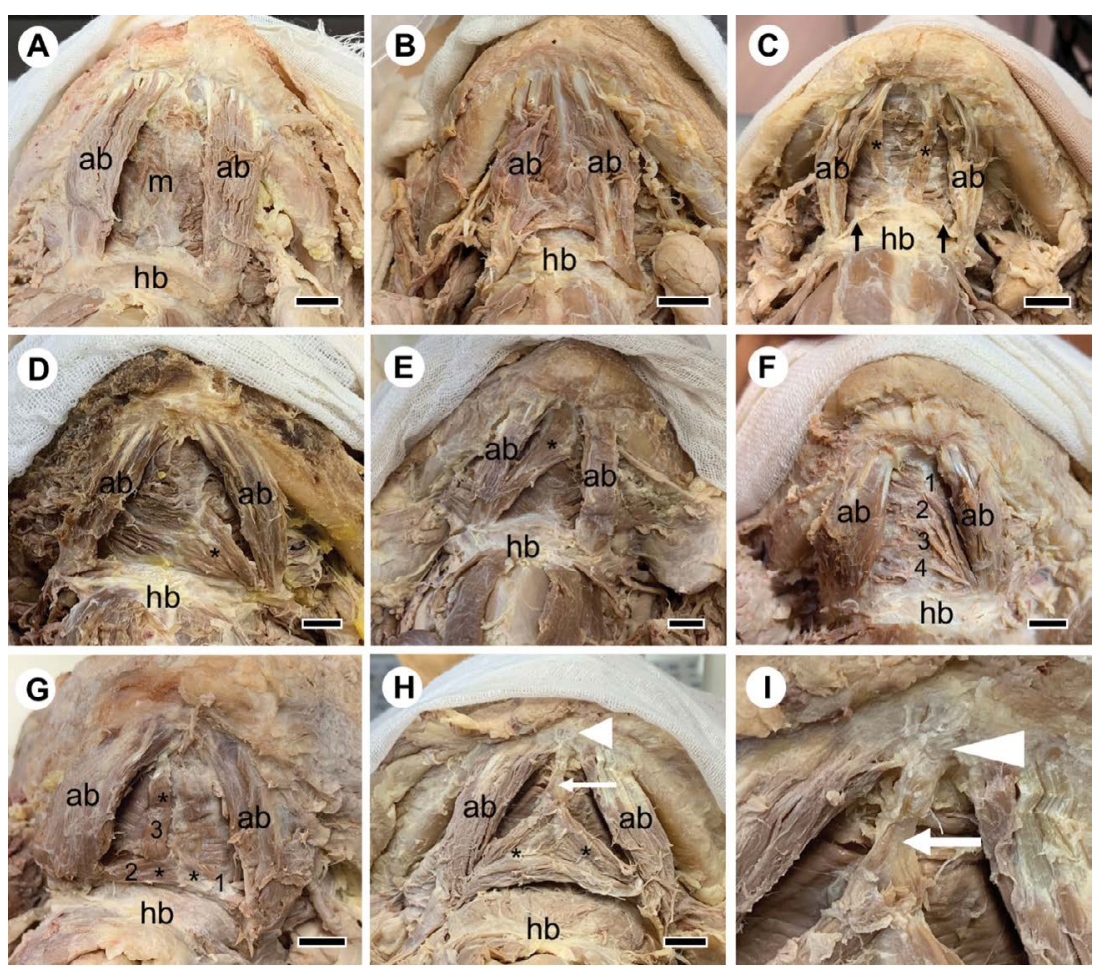

Figure 2. Summary of 1 standard and 8 variations of anterior belly of the digastric muscle (ABDM) observed in the 2019 gross anatomy laboratory. The age of each cadaver and brief description of the observed variations are summarised; $A$. 95-year-old female presenting a standard pattern of the ABDM and the mylohyoid muscle; B. 70-year-old female with fused sheet-like ABDMs. Atavistic type; C. 87-year-old female with a pair of thin anterior belly $(A B)$ accessory muscle strips. Bilateral origin (anterior) type; D. 88-year-old female with an $A B$ accessory muscle on the left side. Unilateral insertion (posterior) type; $E$. 66-year-old female with an $A B$ accessory muscle on the right side. Unilateral insertion (posterior) type; $\mathbf{F}$. 94-year-old female with 4 sprigs of $A B$ accessory muscles on the left side. Unilateral insertion (posterior) type; G. 83-year-old male with $3 A B$ accessory muscles, origin and insertion types on the right and insertion type on the left. Complex type; H. 81-year-old male with bilateral $A B$ accessory muscles. Complex type. Arrow head indicates the ectopic tubercle and arrow indicates the fibrous bundle (origin type variation) connecting the tubercle and the two-insertion type $A B$ accessory muscles; I. A magnified view of the tubercle and fibrous bundle shown in panel $\mathrm{H}$; ab — anterior belly of the digastric muscle; hb - hyoid bone; asterisk — accessory anterior belly muscles; arrows - fibrous tendinous arch. The scale bar corresponds to $1 \mathrm{~cm}$.

examinations of anatomical variations in the submental region, leading to identifying 15 cadavers with non-standard muscle arrangements in the submental region involving the $A B D M$ and the mylohyoid muscle (Figs. 1, 2). Two cadavers (Fig. 1B, C) were also examined further for the innervation and blood supply to the ABDM and its accessory muscle (Fig. 3).

\section{RESULTS}

\section{Structural variations of the submental region muscles}

We have identified anatomical variations comprising atypical arrangements of the ABDM with or without the involvement of the mylohyoid muscle. From 48 cadavers, 15 exhibited submental muscle variations (8 out of 24 cadavers in the year of 2018 and 7 out of 24 cadavers in the year of 2019). Of these 15 cadavers, 8 were male and 7 were female. The average frequency of the bodies with $A B D M$ variations in the 2 years was 31.2\%, and year-to-year frequency was comparable (2018, 33.3\% and 2019, 29.2\%).

In the current study, in an attempt to provide consistency for organizing ABDM variations, we applied the classifications proposed by Yamada (1935) [25] and Zlabek (1933) [28]. Their classification system of non-standard ABDM comprises six types, primarily based on the position(s) of the $A B$ accessory muscles. They are: 1) atavistic type, 2) origin (anterior) type, $3)$ insertion (posterior) type, 4) mixed type, 5) complex (composite) type, and 6) deletion type. In our study, the deletion type, which is characterised by the lack of the ABDM, was not observed. Below, the 5 types of $A B$ variations identified in 15 cadavers in our gross anatomy laboratory sessions are described.

\section{Atavistic type}

The atavistic type ABDM is characterised by its broad attachment at the origin site (mandibular side) 

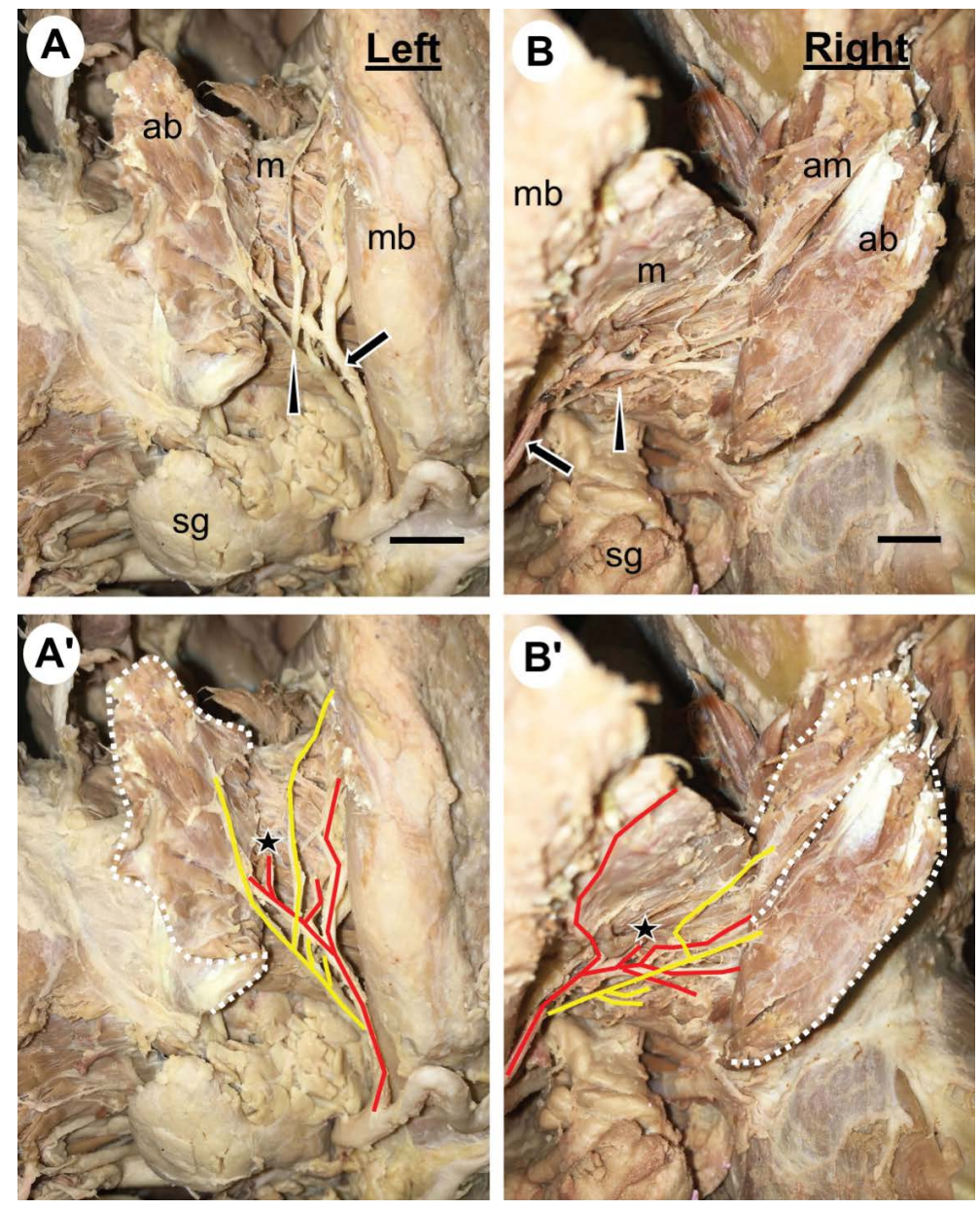

Figure 3. Arterial blood supply and innervation to the anterior belly of the digastric muscle (ABDM) and anterior belly $(A B)$ accessory muscle of the atavistic and origin variations. The submental regions of the atavistic type variation (Fig. 1B) and origin type variation (Fig. 1C) were further dissected to follow the paths of the submental artery and the nerve to mylohyoid. The ABDM and $A B$ accessory muscle were detached from the mandible and reflected to show the arterial supply and innervation; $\mathbf{A}, \mathbf{A}^{\prime}$. The dissection of the left side of the submental region of the cadaver shown in Figure 1B; B, B'. The dissection of the right side of the submental region of the cadaver shown in Figure $1 \mathrm{C}$. In panels $A$ and $B$, black arrow indicates the submental artery and arrowhead indicates the nerve to mylohyoid. In panels $A^{\prime}$ and $B^{\prime}$ the paths of the submental artery and the nerve to mylohyoid are indicated in red and yellow, respectively. The asterisk indicates the location where a branch of the submental artery penetrates the mylohyoid muscle to enter the oral cavity. The white broken line indicates the border of the ABDM and $A B$ accessory muscle; $\mathrm{ab}$ - the anterior belly of the digastric muscle; am - the anterior belly accessory muscle; $\mathrm{m}$ - mylohyoid; $\mathrm{mb}$ - mandible. The scale bar corresponds to $1 \mathrm{~cm}$. as well as the insertion site (hyoid side). The medial borders of the right and left $A B$ muscles touch at the midline to cover the surface of the mylohyoid [25, 28]. The term "atavistic" was proposed because this type of ABDM arrangement has been reported as a standard form in certain non-human primates [4, 22, 25]. In the current study, 2 cadavers exhibited symmetric atavistic type variations (Figs. 1B, 2B). Both of them presented a sheet of $A B D M$, formed by fusion of the right and left anterior bellies. The ABDM sheet and the mylohyoid muscle below were physically separate.

In the atavistic variation shown in Figure 1B, the muscle width at the origin and insertion sites were equally broad and each ABDM presented as a rectangular muscle. Over the mylohyoid raphe the two lower angles of the right and left ABDM rectangular sheets merged, creating a small reverse triangular window below the mandibular symphysis (Fig. 1B). The ABDM attachment on the mandible extended laterally from the digastric fossa and the muscle attachment on the hyoid side extended from the midline on the fibrous tendinous arch towards the intermediate tendon of the digastric muscle. The right and left tendinous arches angled upward and fused at the midline (Fig. 1B). This type of fibrous tendinous arch was initially described by Reid (1886) [16] as "aponeurotic-like tendon" and described also in more recent reports [18, 24]. In the atavistic variation shown in Figure 2B, the medial borders of the right and left ABDMs met at the mandibular symphysis. This positional shift of the attachments resulted in a fully fused ABDM sheet at the midline, forming a symmetric trapezoid muscle sheet overlaying the mylohyoid muscle. In these two atavistic variations (Figs. 1B, 2B), there was no recognizable accessory muscle and the ABDM itself appeared to have broadened to develop a muscle sheet. To the best of our knowledge, these atavistic type variations, in which right and left $A B D M s$ are fused to form a symmetric muscle sheet, have not been reported in the literature.

\section{Origin (anterior) type}

The origin type is characterised by an $A B$ accessory muscle conjoined to an ABDM at the origin (anterior) side that inserts into the fibrous tendinous arch 
attached to the hyoid bone $[25,28]$. This variation may be presented bilaterally or unilaterally. The origin type is further subdivided into the continuous origin type, in which the $A B$ accessory muscle overlaps with the ABDM with no space in-between, and the muscle-bundle origin type, in which the $A B$ accessory muscle is visibly separated from the ABDM [25]. In the current study, we observed 5 muscle-bundle origin type variations; 3 were bilateral (Figs. 1C, D, 2C) and 2 were unilateral (Fig. 1E, F). In the bilateral origin type variations, 2 cadavers presented $2 \mathrm{AB}$ accessory muscles (Figs. $1 \mathrm{C}, 2 \mathrm{C}$ ) and 1 cadaver presented 3 accessory muscles (Fig. 1D). In the variation shown in Figure $1 C$, a robust pair of $A B$ accessory muscles traversed from the mandible to the fibrous tendinous arch. This specific variation likely resembles the variation type previously described as "mento-hyoid" or "m. mentohyoideus" [11, 22] and in a more recent report, as bilateral $A B$ accessory muscles inserting on the fibrous band [24]. The AB accessory muscles observed in the rest of origin type variations were much smaller and thinner. In the bilateral origin type variation shown in Figure 1D, all three accessory muscle strips originated from the medial border of the ABDMs and inserted into the fibrous tendinous arch. In the third bilateral origin type variation (Fig. 2C), the left accessory muscle strip originated from the mandible and inserted into the tendinous arch near the midline, while the right accessory strip originated from the anterior medial border of the ABDM and merged into the mylohyoid muscle. In the two unilateral origin type variations (Fig. 1E, F), accessory muscle strips were found on the right. In the variation shown in Figure 1E, the accessory muscle strip originated from the mandible medial to the digastric fossa and merged with the mylohyoid muscle near the hyoid bone. In the variation shown in Figure 1F, one accessory muscle strip originated from the medial border of the ABDM and the other originated from the mandible medial to the ABDM. Both $A B$ accessory muscles inserted on the hyoid bone.

\section{Insertion (posterior) type}

The insertion type is characterised by the presence of an $A B$ accessory muscle emerging from the intermediate tendon of the digastric muscle medial to the ABDM $[25,28]$. The $A B$ accessory muscle then extends anteromedially to the inter-digastric area. In the majority of cases, an insertion type $A B$ accessory muscle merges with the mylohyoid muscle, most often at the mylohyoid raphe. When there is a space physically separating the $A B$ accessory from the ABDM, the variation is classified as a muscle-bundle insertion type. Alternatively, when the $A B$ accessory muscle overlaps with the ABDM and extends to the mandible, it is classified as the continuous insertion type [25]. In the current study, we observed 5 cadavers presenting unilateral muscle-bundle insertion type variation (Figs. 1G, H, 2D-F). A single AB accessory muscle fanned out from the intermediate tendon medially to merge into the mylohyoid at the posterior half of the raphe (Figs. $1 G, H, 2 D$ ) or at the anterior half of the raphe (Fig. 2E). In one of the insertion type variations, four very delicate sprigs of $A B$ accessory muscles emerged from the intermediate tendon, all of which merged into the mylohyoid muscle at the raphe (Fig. 2F).

\section{Mixed type}

The mixed type is characterised by an $A B$ accessory muscle traversing across the midline in the submental region $[25,28]$. In the current study, we identified 1 cadaver presenting this variation (Fig. 1I). This $A B$ accessory muscle originated from the right side on the mandible and divided into 2 muscle segments on the hyoid side, with the lateral segment inserting on the left digastric intermediate tendon and the medial segment inserting into the mylohyoid muscle at the raphe.

\section{Complex (composite) type}

The complex type is characterised by the coexistence of multiple variations described above, the origin (anterior), insertion (posterior), and mixed types, in one individual $[25,28]$. We identified 2 cadavers presenting bilateral complex type variations (Fig. 2G, H). The complex variation shown in Figure $2 \mathrm{G}$ contained two insertion type $A B$ accessory muscles and one origin type $A B$ accessory muscle. The two insertion type $A B$ accessory muscles were fan shaped, emerging from the right and left intermediate tendons (muscles 1 and 2) and merged with the mylohyoid muscle and with each other at the raphe. The origin type $A B$ accessory muscle (muscle 3 ) emerged from the upper medial border of the ABDM and inserted into the mylohyoid muscle at the superior border of the $A B$ accessory muscle 2 (Fig. 2G). The arrangement of these three $A B$ accessory muscles is similar to the variation reported by Zdilla et al. (2018) [27]. The second complex type variation, shown in Figure $2 \mathrm{H}$, consisted of insertion and origin type variations. How- 
ever, it was unique in the sense that there was an ectopic bony tubercle directly beneath the mandibular symphysis. The presence of this type of ectopic tubercle has not been reported previously. Bundles of tendinous fibres connected the superior angle of the pair of insertion type accessory muscles to this ectopic tubercle (Fig. $2 \mathrm{H}, \mathrm{I}$ ). These fan-shaped $A B$ accessory muscles emerged from the right and left intermediate tendons and merged at the midline with each other and with the mylohyoid muscle at the raphe (Fig. $2 \mathrm{H}$ ).

\section{Arterial blood supply and innervation to the ABDMs and $A B$ accessory muscles}

Arterial blood to the submental region is typically supplied by the submental artery, a branch of the facial artery [6]. Innervation to the anterior belly of the digastric muscle and mylohyoid muscle is typically provided by the nerve to the mylohyoid, a branch of the mandibular division of the trigeminal nerve [12]. To examine whether the arterial blood supply pattern may be altered by the presence of ABDM variations, we dissected the submental region of the 8 cadavers shown in Figure 1B-I and confirmed that the submental artery was the source of the blood supply in all of these cadavers. Innervation to the submental region was also examined in the atavistic type and origin type variations (Fig. 1B, C, respectively). Figure 3 summarises the passages of the submental artery and the nerve to mylohyoid in the submental regions of these cadavers. In the atavistic type ABDM variation (Fig. 3A, $A^{\prime}$ ), the nerve to mylohyoid (indicated by the arrowhead) travelled between the mylohyoid muscle and the ABDM sheet to provide innervation to the both muscles. The submental artery (indicated by the arrow) travelled in parallel with the nerve to mylohyoid and then penetrated the mylohyoid muscle (indicated by the asterisk in Fig. 3A'), entering the oral cavity to supply the sublingual gland. This pattern of the submental artery supplying the sublingual gland is relatively common [7] and it is not associated with particular structural variations of the submental triangle muscles. In the bilateral origin type variation (Fig. 3B, B'), the nerve to mylohyoid also travelled in parallel with the submental artery to pass between the external surface of the mylohyoid muscle and the inner surface of the $A B D M$, and then continued to innervate the $A B$ accessory muscle. Also, in this cadaver, a branch of the submental artery penetrated the mylohyoid muscle to enter the oral cavity to supply the sublingual gland (indicated by the asterisk in Fig. 3B').

\section{DISCUSSION}

The digastric muscle is a suprahyoid muscle that depresses the mandible to assist chewing and speech, and also to stabilise the hyoid bone during swallowing and speaking $[2,20]$. Reflecting the digastric muscle's contribution to these vital human activities, it has been reported that declined volume of the digastric muscle is correlated with the severity of dysphagia in stroke patients [21].

Developmentally, the digastric muscle is a hybrid muscle consisting of two muscle bellies derived from separate embryological origins; the anterior belly originates from the first pharyngeal arch, whereas the posterior belly originates from the second pharyngeal arch. The mylohyoid muscle, located adjacent to the anterior belly muscle, shares a common embryological origin with the ABDM. Accordingly, innervation and blood supply are typically shared between these two muscles, i.e., both are innervated by the nerve to the mylohyoid and supplied by the submental artery, respectively $[14,19]$.

Schematic representations of the types of variations identified in this report, "atavistic, origin, insertion, mixed, complex" are summarised in Figure 4. In the origin type variations, two $A B$ accessory muscles had their distal end fused with the mylohyoid muscle (Figs. 1E, 2C). All of the insertion type $A B$ accessory muscles found in the insertion type only variations (Figs. 1G, H, 2D-F) and in the complex type variations (Fig. $2 \mathrm{G}, \mathrm{H}$ ) consistently had their proximal ends fused with the mylohyoid muscle at the raphe. In the literature, fusion of $A B$ accessory muscle and the mylohyoid has also been repeatedly reported $[8,25$, $28]$. The frequent fusion of the $A B$ accessory muscle and the mylohyoid muscle may indicate that these two muscles develop from a common embryonic primordium during embryogenesis.

In this study, we observed three variations that have not been previously reported in literature. Two are the atavistic type variations (Figs. 1B, 2B) in which right and left $A B D M s$ are fused to form a symmetric muscle sheet. No $A B$ accessory muscles were observed in these variations. The closest variation to these atavistic variations we could find in the literature was a variation in which right and left $A B$ accessory muscles fused at the midline of the submental triangle. In this case, the ABDMs and the accessory muscles were still visibly discernible by distinct striations of the muscle fibres [1]. Another is the complex type variation with an ectopic tubercle elongated from the mandible at 


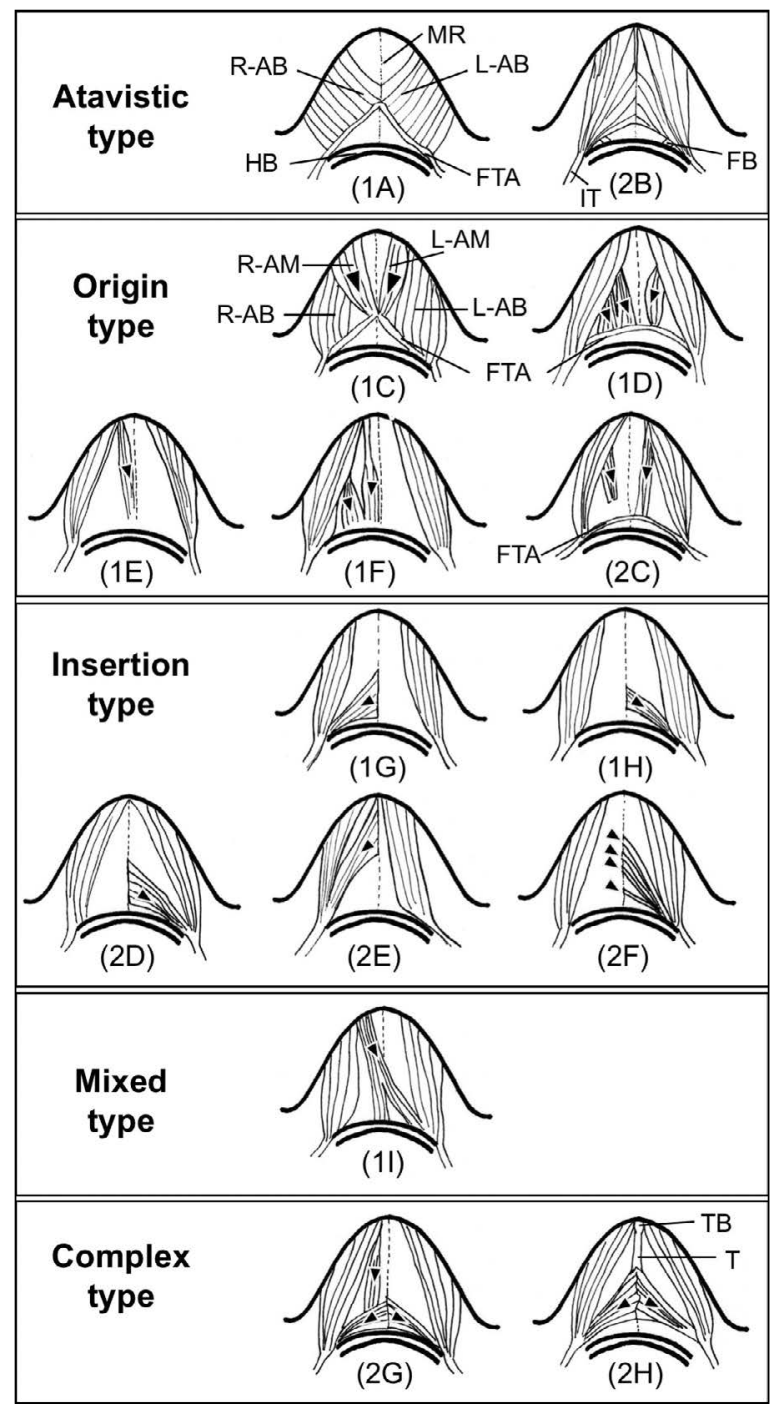

Figure 4. Schematic representations of the anterior belly of the digastric muscle (ABDM) and anterior belly ( $A B)$ accessory muscle variations identified in this report. Sketches highlighting the arrangements of the $A B D M$ and $A B$ accessory muscle variations are shown. Among the 15 variations we have identified, 2 were atavistic type, 5 were origin type, 5 were insertion type, 1 was mixed type and 2 were complex type. Letters in parenthesis shown below the drawings correspond to the listings in Figures 1 and 2 . An arrowhead indicates an $A B$ accessory muscle; FTA - fibrous tendinous arch; $\mathrm{FB}$ - fibrous band; $\mathrm{HB}$ - hyoid bone; IT — intermediate tendon; $R-A B, L-A B$ — right or left anterior belly of the digastric muscle; R-AM, L-AM — right or left $A B$ accessory muscle; $\mathrm{MR}$ - mylohyoid raphe; TB — tubercle; T — tendon.

the symphysis, to which a pair of insertion type $A B$ accessory muscles are attached via a tendinous fibre (Fig. $2 \mathrm{H})$. These three newly identified variations suggest the fluidity of muscle development in the submental region.

Utilising three-dimensional reconstructions of human embryos, it has been reported that the developing mylohyoid and ABDM initially establish an anterior attachment with Meckel's cartilage at the late embryonic stages. While Meckel's cartilage is being encased by the mandibular bone during the late embryonic and early foetal stages, the attachment of these muscles shifts from Meckel's cartilage to the mandibular bone that is extending over the cartilage [15]. Such transitions could lead to erroneous muscle reattachment, possibly causing fragmentation of developing muscles as well as ectopic insertion, which may result in the formation of $A B$ accessory muscles as well as their fusion with the mylohyoid muscle (as described in numerous previous reports) and the $A B$ accessory muscle variations (in the current report). Radlanski et al. (2001) [15] also described that during the early stage of mylohyoid muscle development, some of the muscle fibres gained the direction of striation corresponding to that of developing digastric muscle nearby. This observation may imply a possible developmental process for the origin type $A B$ accessory muscles that are presented in Figure $1 \mathrm{C}$ in this report and also the "mento-hyoid muscle" and " $m$. mentohyoideus" described by Macalister (1875) [11] and Stracker (1908) [22], respectively.

It has been proposed that the relative spatial relationship of an $A B$ accessory muscle with the nerve to mylohyoid, superficial or deep, indicates the origin of muscular primordium from which the $A B$ accessory muscle developed [18]. These authors suggest that the nerve to the mylohyoid penetrates into the middle of the common muscular primordium of the mylohyoid and ABDM during embryogenesis. Consequently, the ABDM develops superficial to, and the mylohyoid muscle develops deep to, the nerve to the mylohyoid. According to this classification system, the sheet-like atavistic type $A B D M$ variation (Fig. 1B) originated from the primordium of the $A B D M$, not from the primordium of the mylohyoid muscle, since it is positioned superficial to the nerve to the mylohyoid (Fig. 3A'). Similarly, the origin type $A B$ accessory muscle shown in Figure $1 C$ originated from the primordium of the ABDM, since it is located superior to the nerve to the mylohyoid (Fig. $3 \mathrm{~B}^{\prime}$ ). Although it is often challenging to dissect intact innervation to small $A B$ accessory muscles, this classification system would be useful to ascertain their embryological origin in relation to the mylohyoid muscle.

Clinically, structural variations of the ABDM have implications in surgeries involving the muscles of the submental region such as correction of facial paralysis and an array of cosmetic surgeries for improving the frontal neck contour $[5,9,10,23,26]$. The ABDM variations, especially those with atavistic type varia- 
tion and robust $A B$ accessory muscles, could cause misidentification of the suprahyoid muscle groups which affects diagnosis involving the submandibular region $[1,26,27]$. Therefore, attracting attention to the frequent occurrence and morphological complexity of ABDM variations in the general population has significant clinical importance.

\section{CONCLUSIONS}

In our current report, we examined 48 cadavers and found that 15 cadavers presented ABDM variations (31.2\%) in the ethnically diverse donor population in Northern California. Since the ABDM is an important landmark for procedures involving the submental region, the high prevalence of non-standard arrangements calls for close attention.

\section{Acknowledgements}

The authors are grateful to the staff of the UC Davis Body Donation Programme for their support and wish to express gratitude to the individuals who donated the bodies for the advancement of medical education and research. The authors declare that they have no conflict of interest to report.

\section{Conflict of interest: None declared}

\section{REFERENCES}

1. Buffoli B, Lancini D, Ferrari M, et al. Symmetrical anatomical variant of the anterior belly of the digastric muscle: clinical implicat. Folia Morphol. 2016; 75(1): 112-116, doi: 10.5603/ FM.a2015.0077, indexed in Pubmed: 26365851.

2. Coquerelle M, Prados-Frutos J, Rojo R, et al. The fetal origin of the human chin. Evol Biol. 2017; 44(3): 295-311, doi: 10.1007/ s11692-017-9408-9.

3. De-Ary-Pires B, Ary-Pires R, Pires-Neto MA. The human digastric muscle: patterns and variations with clinical and surgical correlations. Ann Anat. 2003; 185(5): 471-479, doi: 10.1016/ S0940-9602(03)80110-3, indexed in Pubmed: 14575275.

4. Diogo $R$, Wood B. Soft-tissue anatomy of the primates: phylogenetic analyses based on the muscles of the head, neck, pectoral region and upper limb, with notes on the evolution of these muscles. J Anat. 2011; 219(3): 273-359, doi: 10.1111/j.1469-7580.2011.01403.x, indexed in Pubmed: 21689100.

5. Edgerton MT. Surgical correction of facial paralysis: a plea for better reconstructions. Ann Surg. 1967; 165(6): 985-998, doi: 10.1097/00000658-196706000-00014, indexed in Pubmed: 6026317.

6. Hamparian AM. Blood supply of the human fetal mandible. Am J Anat. 1973; 136(1): 67-75, doi: 10.1002/aja.1001360106, indexed in Pubmed: 4682141.

7. Katsumi Y, Tanaka R, Hayashi T, et al. Variation in arterial supply to the floor of the mouth and assessment of relative hemorrhage risk in implant surgery. Clin Oral Implants Res. 2013; 24(4): 434-440, doi: 10.1111/j.1600-0501.2011.02348.x, indexed in Pubmed: 22092873.

8. Kim SD, Loukas M. Anatomy and variations of digastric muscle. Anat Cell Biol. 2019; 52(1): 1-11, doi: 10.5115/ acb.2019.52.1.1, indexed in Pubmed: 30984445.

9. Labbé D, Giot JP, Kaluzinski E. Submental area rejuvenation by digastric corset: anatomical study and clinical application in 20 cases. Aesthetic Plast Surg. 2013; 37(2): 222-231, doi: 10.1007/s00266-013-0083-7, indexed in Pubmed: 23435506.

10. Langsdon PR, Velargo PA, Rodwell DW, et al. Submental muscular medialization and suspension. Aesthet Surg J. 2013; 33(7): 953-966, doi: 10.1177/1090820X13502220, indexed in Pubmed: 23986490.

11. Macalister A. Additional observations on muscular anomalies in human anatomy. (Third series) With a catalogue of the principal muscular variations hitherto published. Trans Roy Irish Acad Sci. 1875; 25: 1-134.

12. Moore $\mathrm{KL}$, Dalley $\mathrm{AF}$, Agur AMR. Clinically oriented anatomy. 8th ed. Wolters Kluwer, 2018: 1010-1011.

13. Ozgur Z, Govsa F, Celik S, et al. An unreported anatomical finding: unusual insertions of the stylohyoid and digastric muscles. Surg Radiol Anat. 2010; 32(5): 513-517, doi: 10.1007/ s00276-009-0553-4, indexed in Pubmed: 19763380.

14. Pinar YA, Govsa F, Bilge $O$. The anatomical features and surgical usage of the submental artery. Surg Radiol Anat. 2005; 27(3): 201-205, doi: 10.1007/s00276-005-0317-8, indexed in Pubmed: 16003485.

15. Radlanski RJ, Renz H, Tabatabai A. Prenatal development of the muscles in the floor of the mouth in human embryos and fetuses from 6.9 to $76 \mathrm{~mm}$ CRL. Ann Anat. 2001; 183(6): 511-518, doi: 10.1016/S0940-9602(01)80057-1, indexed in Pubmed: 11766522.

16. Reid RW. Anterior belly of the digastric muscle of both sides, arising from the lower border of the horizontal ramus of the inferior maxillary bone. J Anat Physiol. 1886; 21(Pt 1): 78, indexed in Pubmed: 17231671.

17. Sargon MF, Onderoğlu S, Sürücü HS, et al. Anatomic study of complex anomalies of the digastric muscle and review of the literature. Okajimas Folia Anat Jpn. 1999; 75(6): 305-313, doi: 10.2535/ofaj1936.75.6 305, indexed in Pubmed: 10217948.

18. Sakamoto Y, Akita K. Supernumerary muscle bundles in the submental triangle: their positional relationships according to innervation. Surg Radiol Anat. 2004; 26(3): 245-253, doi: 10.1007/s00276-004-0227-1, indexed in Pubmed: 14872289.

19. Som PM, Laitman JT. Embryology, Variations, and Innervations of the Human Neck Muscles. Neurographics. 2017; 7(3): 215-242, doi: 10.3174/ng.3170206.

20. Sowman PF, Flavel SC, McShane $\mathrm{CL}$, et al. Asymmetric activation of motor cortex controlling human anterior digastric muscles during speech and target-directed jaw movements. J Neurophysiol. 2009; 102(1): 159-166, doi: 10.1152/jn.90894.2008, indexed in Pubmed: 19420123.

21. Sporns PB, Muhle $P$, Hanning $U$, et al. Atrophy of swallowing muscles is associated with severity of dysphagia and age in patients with acute stroke. J Am Med Dir Assoc. 2017; 18(7): 635.e1-635.e7, doi: 10.1016/j.jamda.2017.02.002, indexed in Pubmed: 28363443.

22. Stracker O. Die Häufigkeit interponierter Muskelkörper zwischen den vorderen Bäuchen des M. digastricus Anat Anz. 1908; 33: 117-236.

23. Tan ST. Anterior belly of digastric muscle transfer: a useful technique in head and neck surgery. Head Neck. 2002; 24(10): 947-954, doi: 10.1002/hed.10150, indexed in Pubmed: 12369074.

24. Uzun A, Aluclu A, Kavakli A. Bilateral accessory anterior bellies of the digastric muscle and review of the literature. Auris Nasus Larynx. 2001; 28(2): 181-183, doi: 10.1016/s03858146(00)00100-0, indexed in Pubmed: 11240328.

25. Yamada S. Beobachtungen über den Venter anterior des Musculus digastricus mandibulae bei japanishen Erwachsenen und Foeten. Acta Anat Nippon. 1935; 8: 303-347.

26. Zdilla MJ, Pancake AR, Lambert HW. Morphometrics of the anterior belly and intermediate tendon of the digastric muscle: sexual dimorphism and implications for surgery. J Craniofac Surg. 2016; 27(5): 1321-1326, doi: 10.1097/ SCS.0000000000002708, indexed in Pubmed: 27258716.

27. Zdilla MJ, Mangus KR, Swearingen JV, et al. The submental arrowhead variation of the mylohyoid and anterior belly of the digastric muscles. Surg Radiol Anat. 2018; 40(12): 1429-1436, doi: 10.1007/s00276-018-2110-5, indexed in Pubmed: 30306209.

28. Zlabek K. Contribution a la connaissance des anomalies du ventre anterieur du digastrique de l'Homme. Arch Anat Histol Embryol. 1933; 16: 357-406. 